\title{
Kommunikation im Kontext
}

\section{John. J. Gumperz und die Interaktionale Soziolinguistik}

\section{Hubert Knoblauch}

Sozialwissenschaftliche Fakultät, Universität Konstanz, Postfach 5560, D-7750 Konstanz

Zusammenfassung: Die von John J. Gumperz ins Leben gerufene Interaktionale Soziolinguistik ist in der Soziologie bislang ebensowenig rezipiert worden wie ihre Vorläuferin, die Ethnographie der Kommunikation. Deshalb soll gezeigt werden, wie die Untersuchung interkultureller Kommunikation zur entscheidenden Neubestimmung von Konzepten wie Sprachgemeinschaft, Kodewechsel und Diglossie führt; überdies liefert sie - im Sinne einer Hermeneutik des Mißverständnisses - einen wesentlichen Beitrag zur Aufdeckung allgemeiner Strukturen der Kommunikation. Kommunikatives Handeln wird dabei als ein Prozeß der Kontextualisierung verstanden. Verbale und nonverbale Kontextualisierungschlüssel erlauben den Handelnden, auf Intentionen und Situationen zu schließen. Kommunikatives Handeln wird dabei weniger von sprachlichen Mitteln als von interaktiv etablierten Schemata getragen, die als Konventionen sozialer Netzwerke anzusehen sind. Die noch bestehenden Mängel dieses Zugangs können von parallel laufenden Forschungsanstrengungen kompensiert werden, die insgesamt kaum mehr als Sprachsoziologie bezeichnet werden können. Sie bilden vielmehr eine Soziologie der Kommunikation, $\mathbf{d}$. h. der kommunikativen Vorgänge der Wissensvermittlung.

\section{Einleitung}

Die herkömmliche Soziolinguistik und Sprachsoziologie beschäftigt sich mit dem Verhältnis der Sprache zur Gesellschaft im allgemeinen sowie mit der Beziehung zwischen sprachlichen Aspekten menschlichen Handelns und dessen sozialer Organisation. ${ }^{1}$ In Anlehnung an Parsons' Unterstellung, daß Sprachsystem und soziale Gemeinschaften als getrennte Einheiten betrachtet werden könnten, zielte die Forschung auf die „Beziehung“ zwischen sozialen Kategorien (wie etwa „Ethnie“, „Schicht" oder „Geschlecht"), die keinen offensichtlichen Bezug zur Sprache zu haben schienen, und sprachlichen Phänomenen, die unabhängig von der gesellschaftlichen Praxis definiert wurden. Nach einer längeren Phase diachronischer, historisch orientierter Forschung rückte der aktuelle Sprachgebrauch zunehmend in den Mittelpunkt des Interesses, doch selbst in der Soziolinguistik Labov'scher Prägung ${ }^{2}$ wie in der Sprachsoziologie Fishmanscher Provenienz ${ }^{3}$ nimmt Sprache erst dann eine soziale Bedeutung an, wenn sie auf soziale Kategorien bezogen wird, eine Beziehung, die vor allen Dingen in Form von Korrelationen hergestellt wird.

${ }^{1}$ So definiert Hudson (1980: 1) etwa die Soziolinguistik als die Erforschung der Sprache in ihrem Verhältnis zur Gesellschaft; Fishman (1972) sieht den Gegenstand der Sprachsoziologie in der Interaktion zwischen zwei Aspekten menschlichen Verhaltens: dem Gebrauch der Sprache und der sozialen Organisation des Verhaltens.
Diese „korrelationistische“ Sprachsoziologie ${ }^{4}$ stieß spätestens mit Dell Hymes' programmatischem Aufsatz (1962) auf massive Kritik. Hymes wies darauf hin, daß eine solche Vorgehensweise, die Sprache künstlich vom sozialen Handeln abtrennt, den Eindruck erzeugt, als sei die soziale Wirklichkeit außersprachlich und als sei die Sprache nicht sozial. Während Hymes' Standpunkt mittlerweile beinahe in das sprachsoziologische Lehrbuchwissen eingedrungen ist, wurden die von dieser Kritik initiierten empirischen Forschungstraditionen von

${ }^{2}$ Einen wesentlichen Einfluß auf die sich formierende Soziolinguistik hatten zweifellos die Arbeiten von William Labov. Labov versucht zu zeigen, daß sich, über Unterschiede von individuellem Stil und situativem Register hinweg, sprachliche Regelmäßigkeiten für ganze Sprachgemeinschaften finden lassen. Dabei geht er davon aus, daß die Normen als Teil der Sozialisation internalisiert wurden und sich somit automatisch im Verhalten ausdrücken, so daß sozialstrukturelle Variablen mit sprachlichen korrelieren.

${ }^{3}$ Fishman, ein Hauptvertreter der strukturfunktionalistisch orientierten Sprachsoziologie, geht davon aus, $\mathrm{da} ß$ die Regelung zweisprachiger Kommunikation von der Struktur der funktionalen Differenzierung in einer Sprachgemeinschaft, d. h. nach funktionellen „Domänen" wie Schule, Familie, Beruf etc., bestimmt wird.

${ }^{4}$ Trotz gewisser institutionell begründeter Unterschiede verwende ich die Begriffe Sprachsoziologie und Soziolinguistik synonym. Vgl. dazu Blount (1981) und Grimshaw (1987). 
der Soziologie kaum oder gar nicht rezipiert. ${ }^{5}$ Zwar fanden etwa die Ethnomethodologie und die Konversationsanalyse, die diesen Standpunkt teilen, mittlerweile Gehör (Bergmann 1981). Die von Hymes und Gumperz begründete Ethnographie der Kommunikation (auch „Ethnographie des Sprechens") wurde hierzulande von der Soziologie jedoch genausowenig zur Kenntnis genommen ${ }^{6}$ wie die daraus hervorgegangene Kontextanalyse oder die Interaktionale Soziolinguistik (die allein von der Linguistik vereinzelt aufgegriffen wurde). ${ }^{7}$

Die Ethnographie der Kommunikation setzte sich zur Aufgabe, die soziale Prägung der Sprache durch ihre Verortung in Sprechereignissen aufzuzeigen. Vor allem in der Untersuchung sozialer Situationen des Sprechens sollte gezeigt werden, daß der Sprachgebrauch nicht eine Funktion sozialer Strukturen ist, sondern ein für sie konstitutiver Bestandteil. Diese Auffassung wird auch von John Gumperz geteilt, der an der Entwicklung der Ethnographie der Kommunikation maßgeblich beteiligt war. Gumperz und die von ihm begründete Interaktionale Soziolinguistik erweitern diese jedoch durch einen streng interaktionistischen Begriff der Kommuniktion als eines wechselseitigen Wirkhandelns: „Nur wenn eine Handlung eine Reaktion auslöst, können wir sagen, daß kommuniziert wird.“ (1982: 1). Nicht mehr „Sprache und Gesellschaft" sind Gegenstand einer so verstandenen Sprachsoziologie. An die Stelle eines idealisierten Sprachsystems treten kommunikative Prozesse, und an der Stelle der Gesellschaft finden wir „Kontexte“, lokale soziale Strukturen, die durch die kommunikativen Handlungen hervorgebracht werden.

Die Interaktionale Soziolinguistik steuert somit nicht nur Erkenntnisse für sprachsoziologische Sonderprobleme bei. Sie leistet auch einen wesentlichen Beitrag zu einer soziologischen Theorie der Kommunikation. Dabei baut sie zweifellos auf der

\footnotetext{
${ }^{5}$ Die Wegbereiter dieser Entwicklungen, William Labov und Basil Bernstein, stießen hierzulande vor allem in den 70er Jahren weit über die Grenzen der Sprachsoziologie hinaus auf großes Interesse (vgl. Dittmar, 1973).

${ }^{6}$ Eine Rezeption der Ethnographie der Kommunikation beschränkt sich auf wenige Arbeiten, z. B. Luckmann 1986; Coulmas 1979; Arbeitsgruppe Bielefelder Soziologen 1981; Schmitz 1975.

${ }^{7}$ Als jüngstes Beispiel ist sicherlich das linguistische Forschungsprojekt über die Mannheimer Stadtsprache zu erwähnen, das auch Gumperz in seine Arbeiten aufgenommen hat. Vgl. Gumperz (im Druck), Kallmeyer (1990). Vgl. auch Auer (1986).
}

Ethnographie der Kommunikation auf. Deren ungelöster Dichotomie zwischen sozialer Situation und kommunikativen Vorgängen begegnet sie mit dem Interaktions-orientierten Ansatz (II). Ihr empirischer Zugang - die Erforschung interkultureller Kommunikation in modernen Gesellschaften erlaubt eine folgenreiche methodologische Ausweitung des Gegenstands sprachsoziologischer Untersuchungen, da sie den Blick auf die Rolle paralinguistischer und nonverbaler Mittel der Kommunikation eröffnet (III). Daraus resultiert eine Theorie der Kontextualisierung, die kommunikative Vorgänge sprachlicher und nichtsprachlicher Art als integrierte Bestandteile sozialer Situationen und Mittel zur Hervorbringung und Aufrechterhaltung sozialer Strukturen betrachtet (IV). Indem Kommunikation als grundlegend soziales Wirken in Interaktionen angesehen wird, deuten sich auch Lösungswege für das Problem an, wie Interaktionssituationen und gesamtgesellschaftliche Strukturen miteinander verbunden sind und wie sie empirisch untersucht werden können. Die noch bestehenden Mängel dieses Lösungsweges können durch andere Forschungsansätze ausgeglichen werden, die nicht nur dassselbe Ziel verfolgen, sondern auch die Entwicklung zu einer empirischen Soziologie der Kommunikation deutlich machen (V).

\section{Von der Ethnographie der Kommunikation zur interaktionalen Soziolinguistik}

Gegenstand der Ethnographie der Kommunikation ist der Vorgang des Sprechens in sozialen Situationen. ${ }^{8}$ Damit ist auch schon das Ziel um-

\footnotetext{
${ }^{8}$ Die Ethnographie der Kommunikation bzw. des Sprechens nahm ihren Anfang 1962 mit einem Sonderband des American Anthropologist. Der Band beschloß eine Reihe von Symposien, an denen - neben John J. Gumperz und Dell Hymes - Susan Ervin-Tripp, Edward T. Hall, Charles $O$. Frake und William Labov beteiligt waren. Sie weitete sich bald aus und führte zu einer Reihe von Veröffentlichungen, in denen empirisches Material aus den unterschiedlichsten Kulturen vorgestellt wurde. Zu erwähnen sind zweifellos richtungsweisende Sammelbände (Gumperz und Hymes 1972; Bauman und Sherzer 1974, Sanches und Blount 1975), vergleichende Untersuchungen zu einzelnen kommunikativen Bereichen, wie etwa dem der politischen Rhetorik (Bloch 1975; Brenneis und Myers 1984) oder des Streitgespräches (Brenneis 1988) und ehrgeizige Versuche, kommunikative Budgets ganzer Gesellschaften zu erfassen, wie sie etwa von Gossen (1974) und Sherzer (1990) vorgelegt wurden. Eine Zusammenstellung findet sich in Philipsen und Carbaugh (1986)
} 
schrieben. Es besteht darin, den „Sitz im Leben der Sprache" auszumachen, also sprachliche Strukturen so auf Aspekte des Soziallebens zu beziehen, $\mathrm{da} ß$ beide nicht als voneinander isolierte Korrelate erscheinen, sondern $\mathrm{da} \beta$ das Wechselspiel von Sprache, Sprachmustern und sozialer Organisation geklärt wird. Ethnographie der Kommunikation erfaßt einerseits durch intensive Feldarbeit die sozialen Situationen, in denen kommuniziert wird, und andererseits die darin vorkommenden kommunikativen Vorgänge und Strukturen (Hymes 1964: 9).

Diese doppelte Ausrichtung: einerseits sprachlichkommunikative Vorgänge, andererseits soziale Situationen in ihrer gesellschaftlichen Verortung zu untersuchen, führte auch zu einer auffälligen Grenzüberschreitung. Die Beiträge zur Ethnographie der Kommunikation stammen aus Disziplinen wie Volkskunde, Anthropologie, Soziologie, Linguistik, ja sogar der Philologie. Diese Überschreitung herkömmlicher disziplinärer Grenzen zeigt sich an der Bandbreite der gesammelten Daten. Sie reichen von historischen Dokumenten über die materiale Volkskultur und die soziale Organisation bis zum Alltagswissen und zu Einstellungen zur Sprache. Entsprechend vielfältig sind die eingesetzten Methoden: Neben Elizitierungsverfahren und Tests vor allem teilnehmende Beobachtung, Interviews, Interaktions- und Sequenzanalyse sowie linguistische (phonetische, prosodische, lexikalische) Analysen (Saville-Troike 1984). Die Ethnographie der Kommunikation versteht sich als dezidiert induktiv-empirische Disziplin, die sich in starkem Maße auf naturalistische Vorgänge konzentriert. Dieses „naturalistische" Anliegen teilte sie von Anfang an mit Richtungen in der Soziologie, die gemeinhin unter den irreführenden Titel der verstehenden oder "Mikrosoziologie“ gereiht werden. Sprache sollte nicht mehr so behandelt werden, als bestünde ihre ,eigentliche 'Form in einer übersituationalen Grammatik. Sie ist vielmehr ein konstitutiver Bestandteil des sozialen Lebens. Das bedeutete einerseits die Abwendung von intuitiven, formalen Analysen, wie sie die strukturalistische Linguistik und weitgehend auch noch die Sprechakttheorie auszeichneten, und die Zuwendung zu den Situationen, in denen lebende Menschen sprechend handeln. Die Ethnographie der Kommunikaton versteht sich also nicht als „Pragmatik“, die sich neben die Grammatik und Semantik stellt. Vielmehr betrachtet sie die Sprache als lediglich ein Mittel der kommunikativen Interaktion unter anderen. Auf der anderen Seite vermeidet die Ethnographie der Kommunikation, in das vom Strukturfunktionalismus beherrschte Fahrwasser der Sprachsoziologie zu geraten. Anstatt davon auszugehen, daß die Ordnung sprachlicher Vorgänge von der Sozialstruktur determiniert sei (etwa Lautmuster durch Schichten oder Kodewahl durch „Domänen“, d. h. funktional bestimmte Institutionsbereiche), ${ }^{9}$ wurde die Struktur kommunikativer Handlungszusammenhänge als Gegenstand eigenen Rechts untersucht. Typische kommunikative Zusammenhänge werden, in Anlehnung an einen von Roman Jacobson (1960) geprägten Begriff, Sprechereignisse genannt. Das Sprechereignis bildet die Grundeinheit der Untersuchung. Es ist ein zeitlich und räumlich begrenzter sozialer Handlungsablauf, der von Regeln des Sprachgebrauchs beherrscht wird, durch die Ziele, sprachliche Mittel, Teilnehmerrollen und deren Handlungsspielräume festgelegt sind. Hymes' Modell zufolge besteht das Sprechereignis aus einer Reihe von auch nichtsprachlichen „Komponenten", wie etwa Schauplatz bzw. Szene, Teilnehmer oder Personal, Folgen (sowohl Ziele wie Zwecke), Akteigenschaften (Form und Inhalt des Gesprochenen), Stil, Mittel (Kanal und Kode) Normen (der Interaktion und Interpretation) und Genres. ${ }^{10}$ Die Analyse zielt auf die Entdeckung von Regeln, die die Angemessenheit bestimmter Formen des Diskurses in bestimmten Typen sozialer Beziehungen leiten. Mit der Beschreibung der Sprechereignisse sollte der Schritt zur systematischen Erfassung des Sprechhaushalts einer Gesellschaft und seiner Funktionen getan werden.

Zwar waren von Anbeginn Soziologen am Programm der Ethnographie der K'ommunikation beteiligt (Goffman, Sacks, Garfinkel und Schegloff), doch rückte sie institutionell mehr und mehr in die linguistische Anthropologie. Eine Folge dieser anthropologischen Schlagseite war, daß sich eine Vielzahl von Arbeiten auf nichtwestliche, traditionale und lokal begrenzte Gesellschaften konzen-

9 „Domains are defined, regardless of their number, in terms of institutional contexts and their congruent behavioral co-occurrences. They attempt to summarize the major clusters of interaction (...) that occur in clusters in multilingual settings and involving clusters of interlocution." (Fishman 1972).

${ }^{10}$ In einer früheren Version unterschied Hymes sieben Komponenten (Sender, Empfänger, Form der Mitteilung, Kanal, Kode, Thema und Schauplatz) und sieben Funktionen: expressive (emotive), direktive (Konnotation, Pragmatik, Rhetorik ect.), poetische, kontextuelle, metalinguistische, referentielle und Kontakt-Funktionen. 
trieren. ${ }^{11}$ Das gilt weitgehend auch für die aus der Ethnographie der Kommunikaton entstandenen Forschungsrichtungen. Die Arbeiten der „Chicagoer Schule“ (Silverstein, Hanks, Briggs u. a.) sind ausgeprägte Beispiele der anthropologischen Linguistik, die ethnopoetischen Untersuchungen von Hymes beschränken sich vor allen Dingen auf amerikanische Indianer; und die von Hymes und Bauman initiierten, ethnographisch ausgerichteten Performance-Untersuchungen (vgl. Ben-AmosGoldstein 1975) haben in der Soziologie bislang kaum Widerhall gefunden. Eine Ausnahme bildet lediglich die aus der Ethnographie der Kommunikation entstandene Interaktionale Soziolinguistik (Lindenfeld 1984: 132), da sie sich schwerpunktmäßig mit kommunikativen Vorgängen in modernen westlichen Gesellschaften beschäftigt. Überdies wendet sie sich einem Problem zu, das durch den Begriff des Sprechereignisses mehr verdeckt als gelöst wird. Anstatt soziale Situationen durch außersprachliche Komponenten zu bestimmen, stellt sie die Frage: Wie tragen sprachliche Vorgänge zur Herstellung sozialer Situationen bei?

Die von John J. Gumperz ${ }^{12}$ zu Anfang der 70er Jahre ins Leben gerufene Interaktionale Soziolinguistik setzte sich explizit zur Aufgabe, die Dichotomie zwischen sprachlichen Zeichen und sozialem Wissen durch den Vorgang des konversationellen Schließens aufzuheben. Hymes' Auffassung zufolge weist nicht nur das Sprachsystem, die ,langue“, sondern auch die parole selbst, die Produktion und Interpretation von Äußerungen in Interaktionen, Muster auf, die gleichzeitig linguistische und soziale Ausprägungen besitzen. Wie Gumperz (1990b: 2) herausstellt, ist „es die Interaktion selbst, die als unmittelbarste Determinante" der Kommunika-

${ }^{11} \mathrm{Zu}$ den hervorragenden Ausnahmen für Untersuchungen in westlichen Industriegesellschaften gehören zweifellos die Arbeiten von Abrahams (1974) und Kochman (1972) zur Sprechkultur von schwarzen Amerikanern.

12 John J. Gumperz wurde 1922 in Deutschland geboren und kam 1939 in die USA. Er studierte in Cincinnati und graduierte in Chemie an der Universität von Michigan, wo er auch erstmals Linguistik studierte und zur Linguistik überwechselte. 1954, nach zwei Jahren als Dozent an der Cornell University, erhielt er den $\mathrm{Ph}$. D. in Germanischer Linguistik an der Universität von Michigan. Danach folgten bahnbrechende Arbeiten in Südasien. Er ist Professor für linguistische Anthropologie an der Universität Berkeley; zur Zeit forscht er am Max Planck Projekt für kognitive Anthropologie in Berlin. tion fungiert. ${ }^{13}$ Kommunikation, d. h. wechselseitiges, zeichenhaftes Wirkhandeln, ist keine Funktion außersprachlicher Kontexte; vielmehr werden soziale Kontexte mittels kommunikativer Interaktionen hervorgebracht; erst dies ermöglicht Verstehen von Sinn und Bedeutung. Überdies beschränken sich die Mittel zur interaktiven Erzeugung von Kontexten keineswegs auf die Sprache; vielmehr spielen nonverbale Elemente eine wichtige, oftmals entscheidende Rolle in kommunikativen Abläufen. Dazu gehören vor allen Dingen lautliche Mittel; dazu zählen aber auch Mimik, Blick- und Körperbewegungen. Mit diesem interaktionistischen Begriff der Kommunikation richtet sich auch die Interaktionale Soziolinguistik gegen zwei Strukturalismen: gegen den linguistischen Strukturalismus, demzufolge Regelmäßigkeiten der Sprache auf der lexikalischen oder grammatischen Ebene des Sprachsystems zu finden sind, und gegen die strukturfunktionalistische Annahme, daß der Sprachgebrauch von der funktionalen Differenzierung der Sozialstruktur determiniert wird und die Regeln von den Handelnden nur internalisiert zu werden brauchen.

\section{Bilingualismus, Interaktion und die Hermeneutik des Mißverständnisses}

Zur empirischen Erforschung dieses Zieles verbindet die Interaktionale Soziolinguistik verschiedene Analysemethoden: der ethnographische Ansatz wird ergänzt durch ethnomethodologisch bzw. konversationsanalystisch inspirierte Interaktionsanalysen; diese jedoch betonen in einem für soziologische Konversationsanalytiker ungewohnten Maße bislang als „rein linguistisch" angesehene Merkmale, wie Prosodie und paralinguistische Mittel.

Im Mittelpunkt der empirischen Forschung stand von Anbeginn die Untersuchung des Bilingualismus und der interkulturellen Kommunikation (Rehbein 1985). Die Daten wurden in einer Reihe

${ }^{13}$ Alle englischsprachigen Zitate, die im Text vorkommen, wurden vom Autor ins Deutsche übersetzt. Um den Literaturapparat nicht noch mehr zu strapazieren, als dies ohnehin schon der Fall ist, beschränke ich mich auf Gumperz' Arbeiten, ohne auf die Untersuchungen von C. Alvarez-Caccamo, A. Duranti, S. Ervin-Tripp, S. Gal, J. Irvine, M. Jacquemet oder D. Tannen weiter einzugehen. 
unterschiedlichster Gesellschaften erhoben, sie stammen aus Indien, Korea, Österreich, Norwegen, Großbritannien, Mexiko, den Philippinen und den USA. Als Material dienen einerseits ,interne Daten", d. h. Tonband- und Videoaufzeichnungen in den unterschiedlichsten Kontexten (bei sensiblen Kontexten, wie etwa Banken, auch Nachspielungen), und „externe Daten“, d. h. Interviews über Sprechsituationen, Sprechereinschätzung bestimmter Aufnahmen usw. (Ensink 1987).

Gumperz' Forschung läßt sich grob in zwei Phasen aufteilen. Seit Anfang der 60er Jahre führte er Untersuchungen in verschiedenen Sprachgemeinschaften durch, in denen der Schwerpunkt auf dem Verhältnis von linguistischer Variabilität und sozialen Gruppen lag. Obwohl Gumperz schon bei der Begründung der Ethnographie der Kommunikation einer der ersten war, der empirische Studien über sprachliche Interaktionen durchführte, trat dieses Anliegen erst in den späteren Arbeiten in den Vordergrund. An die Stelle der anfänglich untersuchten, deutlich abgegrenzten dörflichen und kleinstädtischen Sprachgemeinschaften rückte mehr und mehr die Untersuchung von Interaktionen, wie sie innerhalb komplexer Gesellschaften zwischen Sprechern aus unterschiedlichen kulturellen Traditionen derselben Gesellschaft stattfanden. Hatten die frühen Untersuchungen einschneidende Folgen für die Vorstellung des Bilingualismus, der Sprachgemeinschaft und des Kodewechsels gezeitigt, so tragen die späteren Untersuchungen nicht nur zur Erhellung der interkulturellen Kommunikation bei; der ,sozial dynamische Begriff des Kontextes" (Gumperz 1991: 41) gibt auch wesentliche Aufschlüsse für die Klärung der Struktur kommunikativer Interaktionen im allgemeinen.

Schon in der frühen Hindi-Urdu Untersuchung zeigte Gumperz, daß Sprachgemeinschaften sich nicht klar voneinander abgrenzen lassen. Die $\mathrm{Re}$ geln für Kodewechsel etwa werden nicht von institutionell bestimmten „Domänen" geleitet, sondern variieren je nach Interaktionssituation. Dadurch kommen ,communities of the mind" in den Blick, die charakteristisch sind für moderne westliche Gesellschaften. Das sind Sprachgemeinschaften, deren Mitglieder sich in den verschiedensten institutionellen und sozialen Bereichen komplexer Gesellschaften finden, die sich aber durch gewohnheitsmäßige Interaktionen, durch die geteilten Sprachvarianten oder sogar durch eine ideologisch motivierte (und zuweilen politisch organisierte)
Treue zur Sprache verbunden wissen. Der Einfluß interaktiver Kontexte auf den Kodewechsel macht es vielmehr nötig, neben den linguistischen Elementen ein zusätzliches soziales Merkmal zu berücksichtigen. Eine Sprachgemeinschaft bezeichnet „jedes menschliche Aggregat (...), das sich durch regelmäßige und häufige Interaktionen auszeichnet, die mittels gemeinsamer sprachlicher Zeichen geführt werden und die sich von ähnlichen Aggregaten durch spezifische Merkmale des Sprachgebrauchs unterscheiden." (Gumperz 1971b: 115). ${ }^{14}$ Sprachgemeinschaften verfügen nicht nur über eine gemeinsame Sprache; sie bilden ein Netzwerk sozialer Interaktionen und sie verfügen über ein gemeinsames Repertoire. Repertoires beinhalten nun nicht mehr nur sprachliche Elemente; um die sie konstituierenden Varietäten zu verwenden, bedarf es zusätzlich eines Gebrauchswissens über soziale Situationen und des darin angebrachten Verhaltens. Repertoire bildet das Sammelbecken für Varietäten. ${ }^{15}$ Anstatt durch eine Sprache, werden Gesellschaften nunmehr durch Repertoires definiert, und die situationsabhängige Verwendung von Varietäten bedeutet, daß Sprecher gewissermaßen ,ein Repertoire sprechen'.

Dieses Verständnis von Sprachgemeinschaften hatte auch Auswirkungen auf die Vorstellungen der Mehrsprachigkeit. Nicht mehr nur der Gebrauch grammatikalisch und lexikalisch unterschiedlicher Sprachen (Hindi oder Urdu, Österreichisch oder Slowenisch, Bokmal oder Ranamal) erwies sich als sozial strukturiert. In der Analyse des Kodewechsels zwischen dem örtlichen Dialekt Ranamal und Bokmal, der Standardsprache, in der norwegischen Kleinstadt Hemnesberget (Blom und Gumperz: 1971a) oder in der Untersuchung slowenischer Minderheiten in Kärnten zeichnete es sich immer deutlicher $a b$, daß die Wahl zwischen Kodes von den jeweils sozial typisierten Kontexten

${ }^{14}$ Diese Auffassung wird von Kloss (1977) heftig kritisiert, der als Alternative den m. E. etwas umständlichen Begriff der Repertoiregemeinschaft anbietet.

15 „A variety is any body of human speech patterns which is sufficiently homogeneous to be analyzed by available technique of synchronic description and which has a sufficiently large repertory of elements and their arrangements of processes with broad enough semantic scopes to function in all normal contexts of communication." (Ferguson und Gumperz 1960: 3). 
des Gebrauchs abhängt (Gumperz 1967, XIII.). ${ }^{16}$ Dabei konnte es etwa im untersuchten indischen Dorf ausschlaggebend sein, ob es sich um eine als persönlich eingeschätzte Interaktion handelte oder ob die Interaktion als „zweckorientiert“ galt; ${ }^{17}$ in anderen Fällen erweist sich die soziale Intention der Sprecher als entscheidend. ${ }^{18}$ Die Unterscheidung typischer Gebrauchssituationen zeigte nicht nur, daß die Sprachgrenzen in zweisprachigen Gesellschaften - auch, wie im Falle von Hindi und Urdu, entgegen den Auffassungen der Sprecher selbst - fließend sind. Überdies führte sie zu einer Ausweitung des Diglossiebegriffs, der nun verschiedene Dialekte, Register oder anders unterscheidbare Varietäten umfaßt, auch wenn sich die Gesellschaft nicht als zweisprachig im engeren Sinn versteht.

In allen Fällen haftet dem Gebrauch eines Kodes eine sozial-symbolische Bedeutung an. Der verwendete Kode dient nicht nur dazu, auf Bedeutungen zu verweisen. Er bringt ebenso Sprecherintentionen, die soziale Identität der Sprecher und den Rahmen der sozialen Situation zum Ausdruck. Die Bedeutung, die im Kodewechsel vermittelt wird, leitet sich ab von einem Zusammenspiel unterschiedlicher kontextueller Elemente: Sprecher, Adressaten, soziale Kategorien, soziale Situation. Dies zeigt sich sehr deutlich beim ,situationalen Kodewechsel", durch den ein anderer Handlungszusammenhang hergestellt (wie etwa vom informellen „Aufwärmen“ zum formalen Interview) werden kann, während beim ,metaphorischen Kodewechsel" der Handlungszusammenhang beibe-

${ }^{16}$ Die soziale Organisation schlägt sich nicht im „Sprachsystem" nieder, sondern vielmehr und direkter im Gebrauch sprachlicher Repertoires, d.h. in den Sprachdistanzen, in grammatischen und lexikalischen Unterschieden zwischen den Sprachen, Dialekten und Sprechstilen. Die Grenzen, die die indische Gesellschaft auszeichnen, spiegelten sich in der Kompartmentalisierung der sprachlichen Interaktion in getrennte kommunikative Sphären wider (J. Das Gupto und Gumperz 1968, 3).

${ }^{17}$ Gumperz (1974: 263) nennt diese Form der Interaktion "transactional“; nur in solchen Situationen konnten Vertreter bestimmter Klassen den regionalen Dialekt und Standard-Hindi verwenden.

${ }^{18}$ Kodewechsel zwischen Englisch und Spanisch beim in Texas verbreiteten TexMex erweist sich als stark kontextgebunden. Englisch wird verwendet, wenn neue Informationen eingeführt werden, während spanisch zur stilistischen Ausgestaltung und zur Verstärkung der Sprecherabsichten eingesetzt wird. Vgl. Gumperz und Hernandez-Chavez (1969: 9). halten wird. Wie die Kontextgebundenheit des Kodewechsels vermuten läßt, ist das Repertoire diejenige Größe, die am engsten mit der Sozialstruktur verknüpft ist. ${ }^{19}$ Selbst die Verwendung derselben Sprache - handele es sich um das Deutsche, Englische oder Amerikanische - weist, je nach Verwendungssituation, systematische Unterschiede auf. Die soziale Funktion des Kodes kann, wie erwähnt, auch von Dialekten, Registern oder Fachsprachen erfüllt werden (Gumperz 1974). Zudem stellte es sich heraus, daß paralinguistische und prosodische Elemente in verschiedenen Sprachgemeinschaften entscheidende, wenn auch je unterschiedliche Funktionen erfüllen. „Parameter wie Geschlecht, Klasse, Ethnie“ sind "nicht selbstverständlich annehmbare Konstanten, sondern kommunikativ produziert" (Gumperz und Cook-Gumperz 1982d: 1). Im Kommunikationsprozeß selbst machen die Sprecher deutlich, welche soziale Identität sie haben, welche Absichten sie verfolgen und in welchen Formen sie dies tun. Die Beschäftigung mit dem Bilingualismus wirft somit die Frage nach einer Theorie auf, „die dem kommunikativen Faktor der sprachlichen Variabilität und ihrer Beziehung zu den Absichten der Sprecher Rechnung trägt, ohne auf unhaltbare funktionalistische Annahmen über Konformität oder Abweichung hinsichtlich eines geschlossenen Normensystems zurückzugreifen (Gumperz 1982b: 29).

Dies erklärt die Verschiebung des Schwerpunkts in der zweiten Phase der empirischen Forschung. Nicht mehr Sprachgemeinschaften, sondern die detaillierte Untersuchung interkultureller Kommunikation steht nun im Mittelpunkt. Nur vordergründig handelt es sich hier noch um die Erforschung des Bilingualismus. Es geht vielmehr darum, die unterschiedlichen Mittel zu bestimmen, die bei Mißverständnissen in Interaktionssituationen zwischen Sprechern aus unterschiedlichen Sprachgemeinschaften zutage treten.

Zwar mag die Untersuchung von interkultureller Kommunikation zunächst als soziolinguistisches Sonderproblem oder als ein lediglich sozialpolitisch motiviertes, gutgemeintes Programm zur Be-

${ }^{19}$ Regeln des Gebrauchs einer Varietät sind geleitet von der sprachlichen Reichweite, d.h. der Distanz zwischen einzelnen Varianten, und dem Grad der Kompartmentalisierung. Sie bilden ein syntagmatisch und paradigmatisch gegliedertes System, das Sprechern rhetorische Optionen zwischen verschiedenen Varietäten eröffnet. Vgl. Gumperz (1970: 13 f). 
seitigung von Kommunikationsstörungen zwischen Handelnden verschiedener Ethnien aufgefaßt werden (vgl. Coulmas 1979; Streeck 1985: 117). ${ }^{20}$ Kommunikationsstörungen und Mißverständnisse sind indessen nicht bloß praktische Handlungsprobleme. Sie bieten vielmehr Musterfälle für die Suche nach Regelmäßigkeiten gelingender Kommunikation. An ihnen offenbart sich in aller Deutlichkeit, wie Kommunikation im Normalfall funktioniert. Sprecher aus unterschiedlichen Kulturen mißverstehen aufgrund vor allem verschieden gebrauchter oder verschieden verstandener paralinguistischer Mittel die Intentionen, die erwartbaren Folgen von Handlungssequenzen und die Tragweite sozialer Situationen. Hier zeigt es sich, daß Handelnde nicht nur mit Sätzen kommunizieren, die Wissen „über“ die Welt vermitteln, „sie stellen ihre Äußerungen zugleich in einen Kontext und ermöglichen so dem Rezipienten Verstehen" (Auer 1986: 23). Bei den ,natürlichen Experimenten“ ähnelnden Felduntersuchungen lassen sich dabei einzelne Faktoren deutlich herausstellen. So beherrschen z. B. viele der in England lebenden Asiaten die englische Sprache perfekt, was Wortschatz und Grammatik angeht. ${ }^{21}$ Dennoch sind Mißverständnisse in Gesprächen mit weißen Engländern die Regel. ${ }^{22}$ Die höfliche Frage des schwarzen Bankangestellten z. B. ist auf eine Weise intoniert, daß sie dem weißen Bankkunden bei-

${ }^{20}$ Einige Texte legen diese zweite Interpretation zweifellos nahe. So bemerken Jupp, Roberts und CookGumperz (1982b: 233): „Wir zeigen hier, wie der in diesem Band vorgestellte Forschungsansatz angewandt werden kann, um Schulungsmaterial und -methoden zu entwickeln, die ein Bewußtsein und eine Toleranz für die Tatsachen und Folgen der linguistischen Unterschiede schaffen kann.

${ }^{21}$ Die Untersuchung der Kommunikation zwischen asiatischen und englischen Bürgern in Großbritannien hat durch einen vom BBC produzierten und ausgestrahlten Lehrfilm einige Bekanntheit erlangt (Gumperz, Jupp und Roberts 1979); mittlerweile sind ihm zwei weitere gefolgt.

${ }^{22}$ Das indische oder pakistanische Englisch nimmt sich in den Ohren von Engländern etwas unbeholfen aus; doch handelt es sich, wie Gumperz zeigt, nicht lediglich um eine stilistische Frage, die sich an dem eigentümlichen und genau bestimmbaren Gebrauch von Pronomen, Relativsätzen und Konjunktionen festmacht. (Eine Reihe dieser Eigenheiten des asiatischen Englisch, wie etwa Wortordnung, der Gebrauch von Verben, Fragepartikeln, Konjunktionen, der Deixis, leiten sich aus den Herkunftssprachen, etwa Hindi, Urdu, ab, die mit dem Englischen Interferenzen, sozusagen Vermischungen, bilden. nahe als ein Befehl erscheint. Und die pakistanische Bedienung in einem Schnellrestaurant im Flughafen Heathrow, die sich in der Intonation ihrer Muttersprache bei ihren Gästen erkundigt, ob sie noch Soße wollen (,gravy?"), wird so verstanden, als wollte sie sagen: „Nun nehmen sie schon endlich die Soße!" (Hinnenkamp 1989: 11).

Hier handelt es sich nicht nur um stilistische Unterschiede. Vielmehr werden durch Betonungen die ,in den Äußerungen ausgedrückten Sprecherabsichten" (Gumperz 1982b: 23) angezeigt. Die bloBe Betonung macht aus der höflichen Frage in der einen Sprachgemeinschaft eine dreiste Aufforderung, in der anderen aus der Bitte einen Befehl, aus der Entschuldigung eine Beschwerde. Diese Mißverständnisse können sich auch in größeren kommunikativen Formaten geltend machen, wie eindringlich in der Fallstudie über einen in den USA der fahrlässigen Tötung angeklagten, philippinischen Arzt geschildert wird. (Gumperz 1982b: 163-194). Obwohl der Arzt flüssig und fehlerfrei amerikanisches Englisch spricht, weist seine Sprache doch auffällige Eigentümlichkeiten auf. Gumperz konzentriert sich hier, wie in vielen seiner Arbeiten, auf den minutiösen Aufweis vor allem paralinguistischer und linguistischer Mittel. Die Wahl von Konjunktionen, Pronomen, aber auch die Markierung zentraler Aussagen (etwa der Handlungen, die der persönlichen Verantwortlichkeit des Arztes zugeschrieben werden sollen) oder beschreibender Passagen werden vom verhörten Arzt auf eine Art getroffen, die sich zum Teil stark von den Sprechgewohnheiten der Vernehmenden unterscheidet. Die Gliederung und Aneinanderreihung von entlastenden Informationen und Evidenzen wird nicht verstanden. Was der Verhörte bezweckt, wird von den weißen Richtern oder FBIAgenten in einzelnen Sequenzen und über weite Strecken des Verhörs nicht mitvollzogen: die Koordination wesentlicher Teile der Interaktion im Verhör mißlingt. Und dies gilt nicht nur für Einzelfälle, sondern für ganze Klassen lebensentscheidender Situationen, wie z.B. Bewerbungsund Bewertungsgesprächen, Sitzungen von Entscheidungsgremien $u$. ä., die eine besondere Strukturierung der Argumentation, eine konventionalisierte Form der Gliederung und spezifische Mittel der Kooperation voraussetzen (Gumperz und Cook-Gumperz, 1982c). Wenn solche Fehlschläge systematisch auftreten, liegen die Ursachen in sprachlichen Gewohnheiten der jeweiligen Sprachgemeinschaften, die gewohnheitsmäßig zum Vollzug bestimmter kommunikativer Zielsetzungen verwendet werden. Das heißt, daß sich die Fehl- 
schläge in den Kommunikationssituationen auf unterschiedliche kommunikative Konventionen zurückführen lassen, mit denen kommunikative Absichten, Formen und Situationen erzeugt werden. ${ }^{23}$ Die Unterschiede lassen sich ,nicht auf blo 3 intentionale Phänomene reduzieren, sie arbeiten gewissermaßen auf der Ebene der Kommunikation selbst" (Gumperz 1970a: 12). ${ }^{24}$

Auf der Negativfolie der mißlingenden Kommunikation lassen sich nun die Umrisse des typischen und routinemäßig funktionierenden kommunikativen Handelns abzeichnen. (Methodisch sichtbar werden die Fehlschläge zunächst an sprachlichen Auffälligkeiten, die zu Störungen im Rhythmus der Kommunikation, zu Konflikten oder Unterbrechungen führen.) ${ }^{25}$ Diese Mißverständnisse nun liegen nicht nur in Unterschieden des Kodes begründet; vielmehr hindern unterschiedliche Konventionen der Kontextualisierung die Beteiligten daran, die Perspektive der anderen zu verstehen und kooperativ Aktivitäten zu vollziehen.

\section{Kontextualisierung der Kommunikation}

Kontextualisierung bedeutet, daß Sprecher und Hörer verbale und nonverbale Zeichen verwenden, mit denen sie das, was zu einer bestimmten Zeit an einem bestimmten Ort gesagt wird, auf Wissen beziehen, das früher angesammelt wurde. Vermöge der Kontextualisierung werden soziale Situationsdefinitionen und Handlungsformen etwa der Wissensvermittlung, Argumentation, Selbstdarstellung ausgehandelt. Sie vollzieht sich 1) innerhalb interaktiver Handlungsabfolgen und 2) vermittels vorläufiger Schlüsse auf die kommunikativen Absichten, die durch 3) typischerweise bekannte Zeichen angedeutet werden.

23 „Konvention“ lehnt sich an Goffmans (1981: 3) Begriff der Ritualisierung an, d. h. teilnehmerspezifische Formen, die sich in einer Gruppierung im und um das Sprechen entwickeln.

${ }^{24}$ Wie Cook-Gumperz (1986) zu zeigen versucht, werden sie als kulturspezifische Konventionen weitgehend schon in der kindlichen Sozialisation gewissermaßen beiläufig erlernt und automatisiert. Vgl. auch SavilleTroike (1984, Kap. 6).

25 „A started sequence is one in which the smooth flow of communication has been disturbed, or there is empirical evidence that a misunderstanding has occurred: Participants show signs of discomfort or annoyance, or otherwise give indications that communication has not been successful.“ (Gumperz und Tannen 1979: 306).
Das Verfahren, durch das verbale und nonverbale Mittel der Kommunikation ,entschlüsselt" werden, nennt Gumperz konversationelles Schließen (,conversational inference“). Damit wird der situative Deutungsprozeß $\beta$ bezeichnet, durch den die Interaktionsteilnehmer die „kommunikativen Intentionen" der je anderen einzuschätzen versuchen, bzw. die ,illokutionäre Kraft" dessen, was mitgeteilt wird und somit als Ausgangspunkt für den nächsten Handlungszug dient.

Die gesprochene Äußerung ist jeweils eine unvollständige Verwirklichung dessen, was in den Köpfen von Sprechern und Hörern ist; vervollständigt wird sie durch das konversationelle Schließen auf verschiedenen Ebenen. Es finden sich Inferenzen, die die einzelnen Handlungszüge und das interaktive Management der Konversation regeln. Konversationelles Schließen zielt auch auf den Rahmen und das Hintergrundwissen darüber, um welche Situation es sich handelt, welche Aufgaben anstehen und welches Verhalten angemessen ist (Gumperz 1990b: 13). Beide Ebenen sind kulturell geprägt: sowohl die Definition und Kategorisierung von Situationen wie die von Mustern der Interaktion und typischen Handlungszielen sind abhängig von der Fähigkeit, Kontextualisierungsschlüssel erkennen und setzen zu können.

Kontextualisierung beruht also auf gemeinsam geteilten Zeichen, Kontextualisierungsschlüsseln ${ }^{26}$. Kontextualisierungsschlüssel sind, bildlich gesprochen, die Knoten im Taschentuch, die den Handelnden bedeuten, was sie tun bzw. wie sie verstanden werden. Die Hörer erschließen den Sinn der Mitteilung und die von Sprechern verfolgten Strategien und Intentionen, indem sie die Schlüssel entziffern, die die Bedeutungen kontextualisieren. ${ }^{27}$ Diese Schlüssel bestehen aus unterschiedlichen Mitteln der Sprachproduktion: Prosodie also Intonation, Betonung, Akzentuierung, ,pitch regi-

${ }^{26}$ „Schlüssel“ trifft m. E. den Sinn der „,contextualization cues“ besser als „Hinweise“" (Streeck 1985; Gumperz 1978), da es sich nicht um gewissermaßen deiktische Verweise handelt, sondern um Schlüsselbart-ähnliche Verbünde, die ein Deutungsschema appräsentieren. Entsprechend wird "inference" als "Schließen" oder „Schlußverfahren" übersetzt.

${ }^{27}$ In einer früheren Arbeit (Gumperz und Herasimchuk, 1975) unterscheidet Gumperz noch explizit Koderegeln, mit denen Gedanken in linguistische Form gebracht werden, von Markierungsregeln, durch die diese Bedeutungen mit rekurrenten Elementen, Vorannahmen und anderen kontextuellen Informationen verknüpft werden. 
ster"; paralinguistische Zeichen: Tempo, Pausen, Verzögerungsphänomene, konversationelle Synchronisierung. ${ }^{28}$ (Zwar wurden prosodische und paralinguistische Zeichen schon sehr detailliert untersucht, doch beschränkte man sich meist auf die Satzebene und auf die Ebene des Ausdrucks; im Mittelpunkt steht hier ihre Rolle für die Herstellung interaktiver Zusammenhänge); Kode-Wahl, d.h. die Wahl zwischen verschiedenen phonetischen, phonologischen und morphosyntaktischen Möglichkeiten, die das jeweilige sprachliche Repertoire anbietet, und schließlich die Ebene des Lexikons und der formularischen Ausdrücke, wie etwa Eröffnungen und Beendigungen, metaphorische Ausdrücke. „Der Wechsel zwischen Kodes, Dialekten und Stilen, prosodischen Erscheinungen (...) wie auch die Wahl aus lexikalischen und syntaktischen Möglichkeiten, formularische Ausdrücke, Gesprächseröffnungen, Beendigungen und Strategien der Sequenzierung können allesamt diese Kontextualisierungsfunktion erfüllen." (Gumperz 1982: 131) Kontextualisierungschlüssel können keinen kontextunabhängigen lexikalischen Bedeutungen zugeordnet werden. Vielmehr sind es mehrere Schlüssel, die gemeinsam in einer typischen Zusammensetzung auftreten und den Schluß auf eine besondere situative Deutung erlauben. ${ }^{29}$ Erscheinen diese Schlüssel den Soziologen zu linguistisch, so wird von Seiten der Linguistik der Einwand erhoben, Gumperz' Vorstellung der Kontextualisierung kranke an einer paralinguistischen Schlagseite (Ensink 1987: 521). Diese Einwände mögen für die meisten von Gumperz' Untersuchungsmethoden ihre Berechtigung haben; sie übersehen aber zum einen eine ganze Serie von Arbeiten zur Kontextualisierung, die andere Methoden verwenden (a), und zum anderen die soziologische Tragweite der Kontextualisierung (b).

(a) Untersuchungen der Kontextualisierung im Rahmen der Interaktionalen Soziolinguistik beschäftigen sich keineswegs nur mit (para)sprachlichen Mitteln. Um etwa die Rolle nonverbaler Schlüssel zu betonen, verweist Gumperz regelmäBig auf die „Kontextanalyse“, die, hierzulande weitgehend unbemerkt, mittlerweile eine eigene

${ }^{28}$ Dazu gehören Tongruppe, Nukleusplazierung und „tune“, d.h. die Richtung der Veränderungen des Intonationsverlaufs im Bereich der Prosodie, sowie Sprechgeschwindigkeit, Lautstärke, Tempo und Rhythmus.

${ }^{29}$ Diese Zusammensetzung läßt sich aus Ko-okkurenzUrteilen ableiten. Cf. Gumperz 1971.
Forschungstradition ausgebildet hat (vgl. Kendon 1990). Sie geht auf die Arbeiten zur nonverbalen Kommunikation zurück, die von Pike und Birdwhistell initiiert und von Scheflen fortgesetzt wurden. Mit den Untersuchungen von Kendon, Erickson, Goodwin und Heath stellten sich zunehmend Überschneidungen mit der Konversationsanalyse ein.

Ziel der Kontextanalyse ist es, „die Einbettung gesprochener Äußerungen in ganze Handlungskonfigurationen" zu untersuchen (Kendon 1990: 3). Methodisch weist sie sich dadurch aus, daß natürliche Interaktionen audiovisuell aufgezeichnet und mit sehr feinen Mitteln sequenzanalytisch angegangen werden. Im Sinne einer empirischen Analyse dessen, was Alfred Schütz die ,Synchronisation der Bewußtseinsströme" nannte, wird die feingliedrige Koordination verbaler und nonverbaler Handlungsabläufe in Interaktionen untersucht. Dabei kann es sich um psychotherapeutische Sitzungen handeln (Scheflen), um Footballspiele (Pike), um das Rauchen einer Zigarette (Birdwhistell), das Blickverhalten beim Küssen (Kendon) oder um Beratungsgespräche in Hochschulen (Erickson und Schultz 1982). Diese Untersuchunnen stellen so etwa heraus, wie Blicksignale und Gesten zur Koordinierung von Redezügen und zur Steuerung der Aufmerksamkeit systematisch eingesetzt werden (Goodwin), wie Blicke und Körperorientierung zur Strukturierung von ArztPatienten-Interaktionen dienen (Heath 1986) oder wie sich Interaktionsthythmen in Familiengesprächen einspielen (Erickson 1982). Während die frühen Arbeiten sich mit allgemeinen Merkmalen der „Feinmechanik der Interaktion“ beschäftigten, wendet sich die Kontextanalyse in zunehmendem Maße den Untersuchungen spezifischer Kontexte, besonders des Arbeitens, zu. Das ist offensichtlich im Falle der von Gumperz bevorzugten ,Gatekeeping"-Situationen. Aber auch die Untersuchung von Leitstellen in Flughäfen (wie sie etwa von der Xerox-Forschungsgruppe in Palo Alto durchgeführt werden), von Leitstellen in U-Bahnen, die von Christian Heath unternommen werden, oder gar des ganzen Dienstleistungsumfelds öffentlicher Verkehrsbetriebe (u.a. bei der RATP in Paris) sind nur einige Beispiele für solche ,studies of work“. ${ }^{30}$ Das spezifische Interesse gilt hier nicht mehr "formalen Strukturen sozialen Handelns", sondern der Spezifik der Kontexte: wie Räumlich-

${ }^{30}$ Auf die Parallelen zur neueren Wissenschaftssoziologie kann hier nicht eingegangen werden. 
keiten, Apparaturen, Zeitbudgets usw, in die Interaktion eingehen, und wie kommunikativ die Arbeitsaufgaben kontrolliert und bewältigt werden.

(b) Grob lassen sich drei verschiedene Kontextebenen unterscheiden, auf die konversationell geschlossen wird: Sprechweisen, Ereignisse bzw. Aktivitäten und soziale Situationen. ${ }^{31}$ Kontextualisierungsschlüssel verweisen zum einen auf Wissen über die Situationen und das darin angemessene Verhalten; zweitens auf die Verfahren der Informations- und Argumentationsstrukturierung; und drittens schließlich auf Sprechweisen, d. h. routinemäßig verwendete linguistische Konventionen, mit denen logische Beziehungen, thematische $\mathrm{Zu}$ sammenhänge und Intentionen angezeigt werden.

Es sind vor allen Dingen „Sprechweisen“, durch die Sprecher anzeigen, welche soziale Identität sie in sozialen Situationen annehmen. Dabei sollte betont werden, daß selbst die ethnische Identität situativ erzeugt wird, wenn z. B. der afroamerikanische Student nach dem Gespräch mit dem weiBen Professor in den „Black artful style“ überwechselt, sobald er sich seinen Kommilitonen zuwendet. Ethnische Identitäten werden durch Sprechweisen angezeigt, die in verschiedenen Situationen sehr unterschiedliche Formen annehmen. Es muß sich keineswegs nur um ethnische Kategorien handeln; fraglos ist, daß auch funktionale Rollen entsprechende kommunikative Routinen erfordern (vgl. Gumperz 1971: 102). Kontextualisierungsschlüssel üben auch eine wichtige Funktion im Vollzug kommunikativer Aktivitäten und in der Strukturierung längerer Sprechereignisse (,events“) aus. Im Unterschied zur Ethnographie der Kommunikation stellt das Ereignis ein mehr oder weniger stark ausgegrenztes Element sozialer Situationen dar, gewissermaßen eine interaktive „Episode“ (Gumperz und Norenz 1990:5), die durch eine spezifische Organisation der Redezüge und der Präferenzen sowie durch thematische und rhythmische Merkmale bestimmbar ist. Ereignisse kreisen um eines oder eine begrenzte Zahl von Themen, und sie lassen sich durch ihre sequentielle Struktur ausgrenzen. Sie zeichnen sich durch typische und somit erkennbare Routinen der

${ }^{31}$ Gumperz selbst wechselt hier zuweilen den Rahmen. In Gumperz (1991) werden drei Ebenen der Allgemeinheit unterschieden, auf denen Kontextualisierungsschlüssel in die Inferenz eingehen: die Wahrnehmungsebene, die Ebene der Sequenzen und die Ebene der Aktivität. Ich beziehe mich hier auf die drei Quellen für das Scheitern interkultureller Kommunikation (Gumperz, Jupp, Roberts: 1979: 5).
Eröffnung und Beendigung aus. ${ }^{32}$ Ereignisse zeitigen soziale Folgen, die Gumperz als Aktivitäten bezeichnet. Aktivität ist gewissermaßen die funktionale Seite des Ereignisses. ${ }^{33}$ Man ist leicht verleitet, Aktivität mit Intention oder Handlungsziel gleichzusetzen. Dieser Vergleich liegt auch nahe, denn Gumperz vergleicht die Aktivität mit dem, was die Sprechakttheorie als illokutionäre Kraft von Äußerungen bezeichnet. Allerdings trifft dieser Vergleich nur bedingt zu. Denn die ,illokutionäre Kraft", von der Gumperz spricht, wohnt nicht einzelnen, isoliert betrachteten Äußerungen inne, sondern interaktiven Sequenzen. ${ }^{34}$

Aktivitäten werden nicht individuell entworfen und spontan koordiniert; vielmehr müssen die Handelnden im jeweiligen Ereignis ein Wissen darüber haben, wie gehandelt werden soll und was damit vollzogen wird. Im interaktiven Vollzug von Ereignissen haben sie immer die Möglichkeit, andere Wege einzuschlagen. Die Ereignisse werden vollzogen, indem die Sprecher ihr ,schematisches Wissen" (Gumperz 1986: 66f) abrufen, das durch die Kontextualisierungsschlüssel angezeigt wird. Solche Schemata können etwa in Frage und Antwort im Verhör, Argumentationen in Versammlungen, Eröffnungen von Verkaufsgesprächen oder ganze Sitzungen, also soziale Situationen sein. ${ }^{35}$ Schemata sind keine statischen Vorgaben, die mechanisch nach Art eines "Situationsskripts" (Streeck 1985: 105) angewandt werden. „Schemata

${ }^{32}$ Elemente des Ereignisses sind eine informative Phrase, d. h. eine semantisch zusammenhängende syntaktische Einheit, die sequentiell eingebettet, rhythmisch abgegrenzt ist und eine prosodisch bestimmbare Konturlinie aufweist sowie von anderen Teilen, etwa durch eine Pause, abgesetzt ist. Gumperz (1990b) unterscheidet darüberhinaus noch Beendigungsphasen, Übergangsphasen zwischen Phrasen usw. Es ist jedoch anzumerken, daß Gumperz zweideutig bleibt, wenn es um das Verhältnis der interaktiv-sequentiellen Organisation zu semantischen, syntaktischen und paralinguistischen Elementen geht.

${ }^{33}$ Aktivität bezeichnet sozial konstituierte, episodenartig-ausgrenzbare Sprechereignisse, die sich durch besondere Schauplätze und Teilnehmer auszeichnen. (Levinson 1979: 367).

${ }^{34}$ Gumperz (1980: 35) kritisiert denn auch, daß sowohl Austin wie Searle die interaktive Seite der kommunikativen Vorgänge übersehen.

${ }^{35}$ Ereignisse müssen nicht notwendig geschlossene Grenzen aufweisen. Vgl. Gumperz und Berenz (1990); dasselbe gilt auch für kommunikative Formen, die unterschiedliche Grade und Ebenen der Verfestigung aufweisen können. 
sind situativ revidierbare, dynamische Strukturen" (Auer 1986: 25). Produktion und Rezeption von Schemata beruhen auf dem gemeinsam geteilten Zugang zu kulturellen Repertoires, die in dem Interaktionszusammenhang, in dem sie vorkommen, eingebettet sind und ihn reflexiv mitgestalten.

Allerdings ist die empirische Bandbreite dessen, was als „Ereignis" bezeichnet wird, sehr breit. Als Ereignis können Frage- und Antwortsequenzen in Interviews gelten wie auch ganze "Jobinterviews". Auf die daraus entstehenden analytischen Schwierigkeiten soll am Ende die Rede kommen. Vorerst sollte der Unterschied zwischen Ereignissen und sozialen Situationen, der nächsten Ebene des Kontextes, hervorgehoben werden. Soziale Situationen bezeichnet, in weitgehender Übereinstimmung mit Goffman, das Wissen um Art, Verhaltensregeln, Rollenmuster, Dekorum, soziale Räume und Zeitstrukturen von Interaktionen, wie etwa bei Bewerbungsgesprächen, Komiteesitzungen usw. „Soziale Situationen bilden den Hintergrund für die Inszenierung einer begrenzten Zahl sozialer Beziehungen im Rahmen eines spezifischen Satzes an Teilnehmerkategorien, d.h. Systemen mit komplementären Verteilungen von Rechten und Pflichten. (Gumperz und Blom 1971) Solche Situationen sind etwa Schulstunden, Seminare, Therapiesitzungen, „Meetings", Verkaufsgespräche. Die Situation enthält einerseits kommunikative Komponenten im engeren Sinn, wie etwa spezifische Diskursformen ("Seminar"). Dazu kommen ethnographische Komponenten, wie Schauplätze (oder „settings“). Schauplatz bezeichnet jene Kategorien, mit denen die Handelnden ihre Umwelt in voneinander unterscheidbare Bereiche unterteilen. Diese Bereiche (,locales") lassen sich durch typische Handlungsmuster und durch ein bestimmtes Personal bzw. „Teilnehmerkategorien" umschreiben.

Die Handelnden besitzen also ein mehr oder weniger umfassendes, großteils ,implizites Wissen" darüber, welche Mittel zur Schaffung und Erhaltung spezifischer Interaktionen, Aktivitäten und Situationen nötig sind. Die Kontextualisierungsschlüssel lenken die für Kommunikation erforderliche Synchronisation der Handelnden und die Koordination von Handlungsteilen in halbwegs verläßliche, bekannte und gewohnte Bahnen. Schemata wie Kontextualisierungsschlüssel sind Teil eines unbefragten, kulturellen Hintergrundwissens, das in den besonderen Handlungssituationen quasi automatisch zur Verfügung steht. Zwar werden die Schlüssel und Schemata aus dem gesellschaftlichen Wissensvorrat der jeweiligen Kultur abgeleitet. Dennoch bewegen sich die Handelnden nicht im Prokrustesbett der vorstrukturierten Kommunikation. Im Prozeß der Erzeugung bestimmter Kontextualisierungsschlüssel und Schemata haben die Handelnden einen Einfluß darauf, auf welchen Bahnen sie sich bewegen sollen. Im Begriff der „Diskursstrategien" kommt zum Ausdruck, daß die Interaktion einem Aushandlungsprozeß (,negotiation") gleichkommt.

Ausgehandelt wird, welches Ereignis und welche Situation die Handelnden hervorbringen. Selbst oder gerade - wenn die Individuen durchaus unterschiedliche Handlungsziele verfolgen, bedarf es dieser Aushandlung auf der Basis einer interaktiven Kooperation. Kooperation heißt nicht notwendig, daß Handelnde an einem, gemeinsamen Handlungsprojekt" arbeiten; um aber zu kommunizieren, müssen Handlungen und Handlungsverläufe aufeinander abgestimmt werden. ${ }^{36}$ Selbst Streitgespräche, ja Kämpfe setzen Kooperation, Aushandlung und ein geteiltes Verständnis der eingesetzten Kontextualisierungsschlüssel und gemeinsamen Schemata voraus. Die Handelnden verwenden somit Diskursstrategien, die zwar $\mathrm{Mu}$ ster vorgeben, die aber nicht ,von außen“ oktroyiert, sondern situativ generiert werden. Die Handlungspläne der Teilnehmer beeinflussen zwar den Kommunikationsproze $\beta$; dies gelingt ihnen aber in Form der Aktivitäten, die sie gemeinsam mit den anderen Beteiligten produzieren. ${ }^{37}$

\section{Kommunikative Kontexte in der Gesellschaft}

Die Interaktionale Soziolinguistik ist also keineswegs nur eine "Theorie des kommunikativen Kodes" (Streeck 1985: 104). Vielmehr versteht sie sich als eine ,soziolinguistische Theorie“, die „allen Aspekten der sprachlichen Kommunikation in menschlichen Gesellschaften" gerecht werden will

36 „In step 1, speakers take in clues from the outside and translate them into appropriate behavioral strategies. This step parallels the perceptual process by referential meanings are converted into sentences. In step 2, these behavioral strategies are in turn translated into appropriate verbal symbols." (Gumperz und Blom 1971: 291).

${ }^{37}$ Diese Abhängigkeit von der interaktiven Kooperation kommt in Goffmans (1981) Begriff der „Co-participants" zum Ausdruck. 
(Gumperz 1990a: 1). „Sprachliche Kommunikation ist deshalb keineswegs bloß ein Vorgang, bei dem die Gedanken in Worte gefaßt werden; sie setzt vielmehr eine aktive Zusammenarbeit zwischen den Produzenten der Mitteilungen und den Rezipienten voraus, deren Antworten als Rückmeldungen für die Schlüsselsituationen der Interaktion fungieren" (Gumperz 1990b: 7). Der Sinn der Handlung, die Bedeutung der Äußerung und ihre Deutung liegt nicht in den Äußerungen selbst, sondern wird im Verlaufe des Austauschs als "kommunikative Intention" konstituiert, die von sprachlichem Wissen, Wissen über Kontexte und Hintergrundannahmen geleitet wird, in denen Deutungen der Hörer mitberücksichtigt sind (Gumperz 1982b: 17). Der ,interaktive“ Kommunikationsbegriff nimmt nicht eine einzelne Sprecheräußerung, sondern einen Austausch oder gar eine Sequenz von Interaktionen zum Ausgangspunkt. ${ }^{38}$ Denn Kommunizieren erfolgt und wird verstanden als Teil einer „kommunikativen Ökolo-

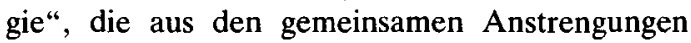
von Sprechern und Hörern besteht.

Handlungstheoretisch überschreitet die so interaktiv verstandene Kommunikation den subjektiven Sinn und führt gewissermaßen ein soziales Eigenleben. Dies kommt nicht nur in Gumperz' Vorstellung der „kommunikativen Intention“ zum Ausdruck, die gewissermaßen ein soziales Faktum für die Beteiligten ist. ${ }^{39}$ Charles Goodwin (1989: 89) zeigt z. B., wie selbst einzelne Äußerungen in ihrer allmählichen Hervorbringung von den Hörern

${ }^{38}$ In Anlehnung an Grice vertritt Gumperz die Auffassung, daß sich eine Grenze zwischen dem Sprechen auf der einen und einem Kern grammatischer und semantischer Merkmale auf der anderen Seite nicht ziehen läßt; vielmehr sind beide Seiten durch die „,konversationelle Implikation", d. h. den dauernden Bezug auf die spezifischen Ereignisse, Aktivitäten und Situationen, miteinander verknüpft.

39 „Beginnen wir erst einmal, Gespräche aus einer interaktiven Perspektive zu betrachten, dann verlassen wir auch den Bereich der individuellen Intentionen und sind gezwungen, geteiltes, gegenseitiges Wissen zu behandeln" (Gumperz 1990b: 13).

${ }^{40}$ D. h. ,daB jeder einzelne Redezug weit davon entfernt ist, das Ergebnis der isolierten Handlung eines einzelnen Individuums zu sein, sondern vielmehr durch die Zusammenarbeit von mehreren Parteien - hier Sprechern und Hörern - konstruiert wird und so eine wesentlich soziale Aktivität ist." Eine Übersicht über Formen und Funktionen verbaler Höreraktivitäten bietet Quasthoff (1981). (durch systematischen Einsatz von Blicken) mitproduziert werden. ${ }^{40}$ Dies gilt nicht nur für wechselseitige Interaktionen, deren Synchronisierung, wie Kendon (1990: 12) betont, durch „transindividuelle Verhaltenssysteme" geregelt wird. William Hanks (1990: 25f) weist darauf hin, daß selbst bei der traditionell vollständig auf das Ego als „Nullpunkt des Koordinatensystems" bezogenen Handlungsform der Deixis „das sprechende Subjekt nie mehr als ein Teil einer sozialen Beziehung" ist. Die Deixis verweist also zunächst nicht „egozentrisch“ auf das Subjekt, sondern ,soziozentrisch" auf die Beziehung zwischen Sprechern und Hörern.

Die zunehmende Eigenständigkeit kommunikativer Strukturen geht einher mit einem Bedeutungszuwachs kommunikativer Vorgänge für die Gesamtgesellschaft. „Die technologische Spezialisierung verkompliziert das Leben auf vielfältige Weise. Doch besondere Aufmerksamkeit verdient die kommunikative Apparatur, die die Gesellschaft schafft, um sichtbar die Rationalität ihrer Entscheidungsprozesse zu demonstrieren" (Gumperz 1982: 4). Dies hat zur Folge, daß kommunikative Vorgänge in größerem Maße über soziale Chancen entscheiden. Die zunehmende Bürokratisierung und Spezialisierung, die immer größere Bereiche des Alltagslebens durchdringt, führt dazu, daß wesentliche biographische Entscheidungen in den unterschiedlichsten Lebensbereichen in Gestalt von kommunikativen Vorgängen gelöst werden, die mehr oder weniger aus dem Handlungszusammenhang ausgekoppelt sind, über den entschieden werden soll. Es bilden sich Filterungen aus, in denen der Zugang zu den unterschiedlichsten Positionen weniger durch deren praktische Ausübung als vielmehr durch kommunikative Fähigkeiten geregelt wird, in „Türstehersituationen" diese Fähigkeiten $z u$ repräsentieren. In Beratungen, Sitzungen, Tagungen, Konferenzen, Versammlungen, Bewertungs- und Bewerbungsgesprächen wird über zentrale organisatorische und persönliche Belange auf der Basis bloßer - und meistenteils unreflektierter - Kommunikation entschieden. „Die Rolle kommunikativer Fähigkeiten in unserer Gesellschaft hat sich deshalb entscheidend verändert. Die Fähigkeit, sich an die unterschiedlichsten kommunikativen Situationen anzupassen und sie zu bewältigen, ist in den Mittelpunkt gerückt, und die Fähigkeit, mit Leuten umzugehen, mit denen man in keinster Weise vertraut ist, wird zur Voraussetzung, um wenigstens über ein Mindestmaß an Kontrolle über die eigene Person und das soziale Umfeld zu verfügen.“ (Gumperz 1982: 4). Funktional spezialisierte Institutionenbereiche werden 
mehr und mehr von kommunikativen Prozessen durchdrungen, in denen relevante soziale Entscheidungen gefällt werden. Die Verankerung der Kommunikation in kulturellen Konventionen führt, im Verein mit der ungleichen Verteilung kommunikativer Kompetenzen, ${ }^{41}$ insbesondere in den zunehmend "multikulturellen" westlichen Gesellschaften zu neuen sozialen Unterschieden. ${ }^{42}$

Diese zunehmende Rolle der Kommunikation wird methodologisch auf zwei Weisen berücksichtigt: (a) Makrostrukturell orientierte Untersuchungen zielen auf soziale Ausprägungen kommunikativer Unterschiede; (b) die ethnographische Vorgehensweise konzentriert sich auf Situationen, in denen sozial folgenreiche Entscheidungen kommunikativ gefällt werden.

(a) Die sozialstrukturelle Zugangsweise betont gesamtgesellschaftliche Machtunterschiede, die in kommunikativen Interaktionen auftreten. Die in der Kommunikation verwendeten sozialen Symbole haben ,nur dann einen Sinn, wenn sie auf die Kräfte bezogen werden, die die Anwendung und Verteilung von Umweltressourcen kontrollieren“" (Gumperz 1982b: 1). In Interaktionen auftretende sprachliche und parasprachliche Muster gelten als „Ausdruck“ für Gruppengemeinsamkeiten, die zur Ausgrenzung gegen andere Gruppen dienen. Anwendung und Verteilung sprachlicher Ressourcen entsprechen den sozialen Ungleichheiten, wie sie von Bourdieu formuliert werden. In Anlehnung an Gramscis Hegemonie-Theorie wird zudem die Bedeutung der Sprache als Ideologie hervorgehoben (Gal 1989). Demnach ist die Sprache eine je nach Verwendungssituation und Sprecherkategorie verschiedene Ausprägung des Habitus. Sie nimmt einen spezifischen Marktwert ein, der sich auf einem Feld mit legitimen und weniger legitimen Varianten streitet. Dieser Konflikt tritt empirisch in Gestalt sprachlicher Interaktionen auf.

${ }^{41}$ In Anlehnung an Hymes beinhaltet kommunikative Kompetenz schon das kulturelle Hintergrundwissen und die Fertigkeiten, Sprache in bestimmten sozialen Situationen zu gebrauchen.

42 „Das Wissen um die Grundzüge sogar auch mancher Formen und Gattungen wechselseitig-unmittelbarer, privater Kommunikation (...), noch stärker das Wissen um halbinstitutionalisierte Kommunikationsweisen, wie z. B. Verkaufs- und Beratungsgespräche, und erst recht das Wissen um hochgradig spezialisierte Fachkommunikation (...) sind zum Bestandteil einer komplexen Wissensverteilung geworden." (Luckmann 1985: 82f).
Im Zusammenhang mit dem Bilingualismus hat diese Lösung schon konkrete Formen angenommen. Vor allen Dingen die soziolinguistische Forschung in Spanien und in Frankreich vertritt eine konflikttheoretische ,Soziolinguistik der Peripherie", die Sprachkonflikte, wie sie in Interaktionen realisiert werden, als Ausdruck von ökonomischen oder Machtkonflikten deutet und sich auf die Aufdeckung des iedologischen Gehaltes kommunikativer Vorgänge konzentriert (vgl. z. B. Irvine 1989; Martin-Jones 1989). ${ }^{43}$

Obwohl diese Zugangsweise von höchster sozialpolitischer Relevanz ist (vgl. Jupp, Roberts, CookGumperz 1982), haften ihr zwei wesentliche Mängel an. Zum einen erscheinen die untersuchten interaktiven Vorgänge lediglich als Aktualisierungen gesamtgesellschaftlicher Konflikte. Das führt wieder in die erwähnte Dichotomie, bei der Erklärungen gleichsam ,von außen“ in die Interaktionen eingeführt und in „Mikroanalysen“ von Kommunikationssituationen bestenfalls bestätigt werden. Sie haben somit methodologisch kaum einen Erklärungswert. Zudem wird diese Vorgehensweise den methodischen Anforderungen an die Interaktionale Soziolinguistik selbst nicht gerecht. Denn ihre Methodik legt ein induktives Verfahren nahe, das auf ,deskriptive Theorien des Sprechens als kulturelles oder als Teil eines kulturellen Systems" zustrebt (Bauman/Sherzer 1975: 98).

In jedem zu erforschenden Handlungsfeld sollte (1) eine intensive, unstrukturierte Ethnographie unternommen werden, die es erlaubt, den Stellenwert einzelner Situationen innerhalb der untersuchten Institutionen, Gruppen oder Organisationen einzuschätzen. Ziel der Ethnographie ist es, Schlüsselsituationen der Kommunikation ausfindig zu machen, d.h. Situationen, in denen wichtige Handlungen der untersuchten sozialen Einheit mit kommunikativen Mitteln vollzogen werden. (2) Diese Situationen werden in einem zweiten Schritt visuell oder audiovisuell aufgezeichnet. (3) Die aufgezeichneten Interaktionen werden sequentiell auf typische Ablaufmuster hin durchsucht und auf spezielle situationsspezifische Merkmale hin analysiert. (4) Schließlich erfolgt ein Vergleich der analysierten Situationen, um systematische Merkmale der kommunikativen Abläufe zu bestimmen.

${ }^{43}$ In Anlehnung an Gumperz hat u. a. Gal (1988) versucht zu zeigen, wie Sprache in der Wolof-Gesellschaft durch die politische Ökonomie geregelt wird, d. h. als eine Form des kulturellen Kapitals fungiert, das zur Aufrechterhaltung und Legitimation von Ungleichheiten und Hegemonien dient. 
(b) Dieser deskriptiv-ethnographische Zugang geht davon aus, daß beobachtbare Phänomene, wie Interaktionen und Situationen, nicht aus hochgradig theoretischen und meist nur metaphorisch verstehbaren sozialstrukturellen Kategorien abgeleitet werden dürfen. Mit der ethnographischen Vorgehensweise steht die Interaktionale Soziolinguistik vor einem Problem, das vielleicht Goffman (1983) am deutlichsten formuliert hat: Auf welche Weise lassen sich durch Untersuchungen flüchtiger Interaktionssituationen und der von ihnen erzeugten Kontexte Schlüsse auf die soziale Struktur ziehen? Oder, um mit Gumperz zu fragen, wie kommt die Sozialstruktur in die Interaktion? ${ }^{44}$

Einen Lösungsweg eröffnet die Forschungsstrategie der Ethnographie von Türsteher- bzw. Schleusensituationen. Gegenstand der Interaktionalen Soziolinguistik sind kommunikative Vorgänge an Bankschaltern, in Bewertungs- und Bewerbungsgesprächen, im Schulunterricht, Aufnahmegespräche zur beruflichen Weiterbildung, Diskussionen und Komiteesitzungen, politische Reden, Werbung usw. All den Untersuchungen ist gemeinsam, $\mathrm{da}$ sie gesellschaftlich relevante Handlungsfelder, also Schlüsselsituationen, so naturalistisch wie möglich zu erfassen versuchen. Hinter der Auswahl solcher Situationen steht die Annahme, daß innerhalb der verschiedenartigsten Organisationen sozial folgenreiche Entscheidungen (Aktivitäten) immer häufiger mittels rein kommunikativer Vorgänge (Ereignisse) gefällt werden. In Kommunikationssituationen dieser Art berühren sich Kommunikation und Sozialstruktur gewissermaßen unmittelbar. Weisen schon die sozialen Folgen kommunikativer Schlüsselsituationen über die Situation hinaus, so wird der weitere ethnographische Kontext mit einem zweiten Begriff zu fassen versucht.

Die Art der sozialen Situationen und die Weisen ihrer Bewältigung unterscheiden sich je nach sozialen Netzwerken, die sich durch jeweils besondere kommunikative Konventionen auszeichnen. ${ }^{45}$ Netzwerke, die als Suchbegriffe vor allem der urbanen Anthropologie seit den 60er Jahren dienten, werden von Gumperz als institutionalisierte zwi-

${ }^{44}$ Es handelt sich hier um den Zusammenhang zwischen "mikrosoziologischen“ Interaktionen und "makrosoziologischen Phänomenen (vgl. Grimshaw 1987a).

${ }^{45}$ Einen frühen Versuch, eine makrostrukturelle Beschreibungskategorie zu finden, stellt die „Kodematrix" dar, die Verteilung funktional spezialisierter Kodes, die an sozialen Rollen orientiert ist (Gumperz 1971: 102). schenmenschliche Beziehungen angesehen, in denen regelmäßig mit Blick auf bestimmte Ziele kommuniziert wird. Während etwa Milroy ${ }^{46} \mathrm{zu}$ einer quantitativen Definition von Netzwerken neigt, betont Gumperz die Qualität der institutionalisierten Typen der Interaktion und ihrer sprachlichen Ausprägung. Von Netzwerken kann gesprochen werden, wenn Individuen regelmäßig mit anderen im Rahmen einer bestimmten Aktivität handeln, wodurch sich konventionalisierte Beziehungen und Erwartungen über wechselseitige Rechte und Pflichten ergeben. ${ }^{47}$ Netzwerke werden nicht durch „soziale Strukturen“ definiert; vielmehr sind sie durch die kommunikativen Handlungen und Formen bestimmt, die es auch ermöglichen, Netzwerktypen $\mathrm{zu}$ unterscheiden (Gumperz und Tannen 1979: 308, Gumperz und Blom 1971a).

Ereignis, Schlüsselsituation und Netzwerk zeichnen zwar die Richtung vor, die eine deskriptivethnographische Erforschung von Kommunikation im Kontext nimmt. Dennoch dürfen kritische Schwachpunkte der Interaktionalen Soziolinguistik nicht übersehen werden.

Aus soziologischer Sicht fällt schon der Begriff des Netzwerks zu ungenau aus. Es dürfte nötig sein, Beschreibungskategorien zu entwickeln, die zwischen Netzwerken und sozialen Situationen vermitteln, wie etwa zeitlich und räumlich begrenzte soziale Milieus. ${ }^{48}$. Es dürfte nötig sein, die analyti-

${ }^{46}$ Milroy (1987) zeigt an drei Belfaster Unterschichtsgruppen, daß nicht die Zugehörigkeit zu einer sozialen Schicht, sondern zu einem sozialen Netzwerk entscheidend für Unterschiede im Sprachgebrauch ist. Netzwerke unterscheidet er nach der Häufigkeit und der Frequenz sozialer Beziehungen und nach den entsprechenden sprachlichen Ausprägungen.

${ }^{47}$ "Wir können zeigen, daß der Sprachgebrauch von Individuen tatsächlich Muster aufweist und daß, zweitens, die Fähigkeit, von diesen Mustern Gebrauch zu machen, wesentlich dafür verantwortlich ist, daß das Individuum an den Beziehungsnetzwerken teilhat und schließlich auch an den sozialen Gruppierungen, die die soziale Welt ausmachen" (Gumperz 1990a).

${ }^{48}$ Grathoff (1989) definiert soziale Milieus als zeitlich und räumlich ausgegrenzte soziale Einheiten, die sich durch feste und dauerhafte Sozialbeziehungen, gewohnheitsmäßige Orte der Interaktion und Kommunikation, gemeinsame Zeitbudgets und eine gemeinsame "Geschichte" auszeichnen." Dabei kann es sich z. B. um Familien handeln oder etwa um Betriebe, Vereine und andere Organisationen. Wie etwa Jones (1987) zeigt, bilden selbst Organisationsmilieus spezifische kommunikative Kulturen bzw. eine „organizational folklore" aus. 
sche Begrifflichkeit der Ethnographie der Schlüsselsituationen zu verfeinern. ${ }^{49}$ Situationen, in denen über den Zugang von Personen entschieden wird (die von Gumperz bevorzugt werden, weil hier soziale Kategorien der Person thematisch werden), unterscheiden sich von solchen, in denen nicht über Personen, sondern über sozial folgenreiche gemeinsame Handlungspläne entschieden wird. Überdies machen die Ausführungen zur Kontextanalyse deutlich, daß die Rolle prosodischer Kontextualisierungsschlüssel überschätzt wird. Auch die Analyse längerer kommunikativer Abläufe leidet an einer begrifflichen Unschärfe, wenn lediglich $z$ wischen ,argumentativen" oder „informativen“ Typen unterschieden wird. Und schließlich haftet dem Ereignis eine zu große Ambivalenz an, umfaßt es doch Handlungen verschiedenster Spannweiten - von Bitten bis hin zu ganzen Interviews.

Gerade diese Schwachstellen verweisen jedoch auf Parallelen in anderen Forschungstraditionen, die gewissermaßen komplementär dasselbe Ziel verfolgen. $\mathrm{Zu}$ erwähnen ist hier nicht nur die MilieuAnalyse und die Konversationsanalyse, die auch sprachliche und parasprachliche Mittel der Kontextualisierung erforscht und mit der die Interaktionale Soziolinguistik ohnehin in engem Kontakt steht. Die Analyse längerer kommunikativer Ereignisse, wie etwa Argumentationen oder Rekonstruktionen, wird von der Gattungsforschung (Luckmann 1986) weitergeführt, die es erlaubt, zwischen Abläufen unterschiedlicher Verfestigungsgrade und der verschiedensten Spannweiten zu unterscheiden. Zudem ermöglicht sie es, eine Verbindung zur sozialen Struktur der Produzenten herzustellen, wie etwa Hanks (1987) am Beispiel von Gattungen der politischen Kommunikation oder Gal (1990) für den Zusammenhang von geschlechtsspezifischen Gattungen und Machtunterschieden zeigen. Die Komplementarität der ethnographisch orientierten Forschungsanstrengungen offenbart sich schließlich auch am gemeinsamen Ziel: durch die empirische Erforschung des „kommunikativen Haushalts" (Luckmann 1986) bzw. der "kommunikativen Ökonomie“ (Gumperz 1982, 43) sollen die kommunikativen Prozesse erfaßt werden, in denen gesellschaftlich relevantes Wissen vermittelt wird; dadurch soll Aufschluß

${ }^{49}$ Wie sich in Gumperz grober Unterscheidung von „transaktionalen“ und ,persönlichen" Interaktionen zeigt, umfassen soziale Netzwerke jedoch oft verschiedene Bündel sozialer Situationen vor allem über institutionelle Zusammenhänge hinweg. über das tatsächlich wirksame „Relevanzsystem“ bzw. über die „Valorizations" gewonnen werden.

So lückenhaft der „ethnographische“ Zugang auch noch sein mag, scheint es doch deutlich, wie sich in verschiedenen Disziplinen ein Ansatz zur Erforschung kommunikativer Prozesse der Wissensvermittlung entwickelt, der keineswegs mehr in die klassische „Sprachsoziologie“ fällt. Indem diese Soziologie der Komunikation den Zugang zum sozialen Handeln über die empirisch faßbaren kommunikativen Konstruktionen sucht (Schütze 1987), trägt sie entscheidend zur „kommunikativen Wende" der Kultur- und Wissenssoziologie bei. Anstatt Kultur von der Sozialstruktur zu isolieren, rücken die kommunikativen Vorgänge der gesellschaftlichen Wissensproduktion und -vermittlung in den Vordergrund, in denen soziale Strukturen mittels kultureller Muster geschaffen und aufrechterhalten werden. Durch die Theorie der Kontextualisierung zeigt die Interaktionale Soziolinguistik, wie die Verbindung zwischen kommunikativen Situationen und Sozialstruktur hergestellt wird; sie macht auch deutlich, wie die Theorie des kommunikativen Handelns mit einer empirischen Forschung verbunden werden kann, die sowohl der gesellschaftlichen Entwicklung Rechnung trägt und gleichwohl nicht auf die Perspektive der Handelnden verzichtet.

\section{Literatur}

Abrahams, R. D., 1974: Black talking on the streets, S. 240-262. In: Baumann/Sherzer, 1974.

Ammon, U./N. Dittmar/ K. J. Mattheier (Hrsg.), 1987: Sociolinguistics - Soziolinguistik. Ein internationales Handbuch zur Wissenschaft von Sprache und Gesellschaft. 1. Halbband. Berlin und New York: De Gruyter.

Arbeitsgruppe Bielefelder Soziologen (Hrsg.), 1981: Alltagswissen, Interaktion und gesellschaftliche Wirklichkeit. Opladen: Westdeutscher Verlag (5. Aufl.).

Auer, P., 1986: Kontextualisierung. Studium Linguistik $19,22-47$.

Bauman, R./ J. Sherzer (Hrsg.), 1974: Explorations in the Ethnography of Speaking. Cambridge University Press.

Bauman, R./ J. Sherzer, 1975: The ethnography of speaking. Annual Review of Antrophology, Band 4, 95-119.

Ben-Amos, D./ K. S. Goldstein (Hrsg.), 1975: Folklore: Performance and Communication. Den Haag: Mouton.

Bergmann, J., Ethnomethodologische Konversationsanalyse, in: P. Schröder/ H. Steger (Hrsg.), Dialogforschung. Düsseldorf: Schwann. 
Bloch, M. (Hrsg.), 1975: Political Language and Oratory in Traditional Societies. London: Academic.

Blount, Ben, G., 1981: Sociolinguistic Theory in Anthropology. International Journal for the Sociology of Language 31: 91-108.

Brenneis, D, 1988: Language and Disputing. Annual Review of Anthropology 17: 221-237.

Brenneis, D. L./ F. R. Myers, (Hrsg.), 1984: Dangerous Words: Language and Politics in the Pacific. New York University Press.

Cook-Gumperz, J., (Hrsg.), 1986: The Social Construction of Literacy. Cambridge University Press.

Coulmas, F., 1979: Einleitung, in: Dell Hymes, Soziolinguistik. Zur Ethnographie der Kommunikation. Frankfurt.

Dittmar, N., 1973: Soziolinguistik, Frankfurt am Main.

Ensink, T., 1987: Interpretative Processes in Discourse, The Approach by John Gumperz. Journal of Pragmatics 11: $517-531$.

Erickson, F./ J. Schultz, 1982: The Counsellor as Gatekeeper: Social Interaction in Interviews. New York.

Erickson, F., 1982: Money Tree, Lasagna Bush, Salt and Pepper: Social construction of topical cohesion in a conversation among Italian-Americans, in: $\mathrm{D}$. Tannen (Hrsg.), Analyzing Discourse: Text and Talk. Washington D. C.

Ferguson, C. A./ J. J. Gumperz, 1960: Linguistic diversity in South Asia. Studies in regional, social, and functional varieties. International Journal of American Linguistics Vol. $26 \mathrm{Nr} .3$.

Fishman, J. A., 1972: The Sociology of Language: An Interdisciplinary Social Sciences Approach to Language in Society. Rowley/Mass.

Gal, S., 1988: The political economy of code choice, in: M. Heller (Hrsg.), Codeswitching: Anthropological and Sociological Perspectives. Berlin.

Gal, S., 1989: Language and Political Economy. American Anthropologist 18, 345-367.

Gal, S., 1990: Between speech and silence: The problematics of research on language and gender, in: $M$. DiLeonardo (Hrsg.), Toward a New Anthropology of Gender. Berkeley: University of California Press.

Goffman, E., 1983: The interaction order. American Sociological Review 48: 1-17.

Goffman, E., 1981, Forms of Talk. Oxford: Blackwell.

Goodwin, C., 1989: Turn construction and conversational organization, S. 88-102. In: B. Dervin, L. Grossberg, B. O'Keefe und E. Wartella (Hrsg.), Rethinking Communication. Band 2: Paradigm Exemplars. Newbury Park, London Neu Dehli.

Gossen, G. H., 1974: Chamulas in the World of the Sun: Time and Speech in Maya Oral Tradition. Cambridge/ Mass.: HUP.

Grathoff, R., 1989: Milieu und Lebenswelt. Frankfurt: Suhrkamp.

Grimshaw, A. D., 1987: Micro-Macrolevels, S. 66-77. In: Ammon/Dittmar/Mattheier (Hrsg.).

Grimshaw, A. D., 1987a: Sociolinguistics versus Sociology of Language: Tempest in a Teapot or Profound Academic Conundrum?, S. 9-16. In: Ammon/Dittmar/Mattheier (Hrsg.).
Gumperz, J. J., 1961: Speech variation and the study of Indian civilization. American Anthropologist 63: 967-988.

Gumperz, J. J., 1967a: Introduction, in: ders. und June Rumery, Conversational Hindi-Urdu. Band 1, NeuDehli.

Gumperz, J. J./J. Das Gupto, 1968: Language, communication and control in North India. Berkeley, Language Behavior Research Laboratory.

Gumperz, J. J./E. Hernandez-Chavez (1969): Cognitive aspects of bilingual communication. Berkeley, Language Behavior Research Laboratory.

Gumperz, J. J., 1970: Sociolinguistics and communication in small groups. Berkeley, Language Behavior Research Laboratory.

Gumperz, J. J., 1970a: Verbal strategies in multilingual communication, S. 129-147. In: J. Alatis (Hrsg.), Report of the 21. Annual Round-Table Meeting on Linguistics and Language Studies. Washington D. C.

Gumperz, J. J., 1971: Language in Social Groups. Essays by John J. Gumperz. ausgew. u. eingel. v. A. S. Dil. Stanford.

Gumperz, J. J./ J.-P. Blom, 1971a: Social meaning in linguistic structures: Codeswitching in Norway, $\mathbf{S}$. 251-310. In: Gumperz, 1971.

Gumperz, J. J., 1971b: The speech community, in Gumperz, 1971.

Gumperz, J. J./ D. Hymes (Hrsg.), 1972: Directions in Sociolinguistics: The Ethnography of Communication. New York.

Gumperz, J. J., 1974: Linguistic and social interaction in two communities, in: B. G. Blount (Hrsg.), Language, Culture and Society. Cambridge/Mass.

Gumperz, J. J./ E. Heramsichuk, 1975: The conversational analysis of social meaning: A study of classroom interaction, S. 81-115. In: Sanches/ Blount (Hrsg.).

Gumperz, J. J., 1978: Sprache, soziales Wissen und interpersonale Beziehungen, S. 114-127. In: U. M. Quasthoff (Hrsg.), Sprachstruktur - Sozialstruktur. Zur linguistischen Theoriebildung. Königstein/Ts.

Gumperz, J. J./ D. Tannen, 1979: Individual and social differences in language use. In: C. Fillmore, D. Kempler und W. S. Y. Wang (Hrsg.), Individual Differences in Language Ability and Language Behavior. New York.

Gumperz, J. J./ T. C. Jupp/ C. Roberts, 1979: Crosstalk. A Study of Crosscultural Communication. Background Materials and Notes of the BBC film. Southall.

Gumperz, J. J., 1980: The sociolinguistic basis of speech act theory, in: J. Boyd und S. Ferrara (Hrsg.), Speech Act Theory Ten Years After. Mailand.

Gumperz, J. J., 1982: Discourse Strategies. Cambridge University Press.

Gumperz, J. J. (Hrsg.), 1982b: Language and Social Identity. Cambridge University Press.

Gumperz, J. J./ Jenny Cook-Gumperz, 1982c: Interethnic communication in committee negotiations, $\mathrm{S}$. 145-162. In: Gumperz (1982b).

Gumperz, J. J./ Jenny Cook-Gumperz, 1982d: Introduction, S. 1-21. In: Gumperz (1982b). 
Gumperz, J. J., 1986: Interactional sociolinguistics in the study of schooling, S. 45-68. In: Cook-Gumperz (Hrsg.).

Gumperz, J. J./ Norine Berenz, 1990: Transcribing conversational exchanges, in: M. D. Lambert (Hrsg.), Transcribing and Coding Methods for Language Research. Hillsdale.

Gumperz, J. J., 1990a: Linguistic variability in interactional perspective, in: W. Kallmeyer (Hrsg.).

Gumperz, J. J., 1990b: Speech community in interactional perspective, Vortrag bei der 2. Konferenz der International Pragmatics Association, Barcelona, 9. - -13. Juli.

Gumperz, J. J., 1991: Contextualization and Understanding, in A. Duranti und C. Goodwin (Hrsg.), Rethinking Context. New York: Cambridge University Press.

Gumperz, J. J. (im Druck): Theory and method in pluriglossia: the interpretive analysis of language use, in: $\mathrm{R}$. Gusmani (Hrsg.), Aspetti metologici e teorici nelle ricerche sul plurilinguismo nei territori del' AlpeAdria. Udine.

Hanks, W. F, 1987: Discourse Genres in a Theory of Practice. American Ethnologist 4/14: 668-692.

Hanks, W. F., 1990: Referential Practice. Cambridge University Press.

Heath, C., 1986: Body Movement and Speech in Medical Interaction. Cambridge.

Hinnenkamp, V. (1989): Interaktionale Soziolinguistik und interkulturelle Kommunikation. Tübingen.

Hudson, R. A., 1980: Sociolinguistics. New York: CUP.

Hymes, D., 1962: The ethnography of speaking, in: Th. Gladwin und W. Sturtevant (Hrsg.), Anthropology and Human Behavior. Washington.

Hymes, D., 1964: Introduction: Toward ethnographies of communication, S. 1-34. In ders.

Hymes, D./ J. Gumperz, 1964: The Ethnography of Communication. American Anthropologist 4: 1.

Hymes, D., 1972: Models of the interaction of language and social life, S. 35-71. In: Gumperz/Hymes, 1972.

Hymes, D., 1979: Soziolinguistik. Zur Ethnographie der Kommunikation. Frankfurt: Suhrkamp.

Irvine, J., 1989: When talk isn't cheap: Language and political economy. American Ethnologist 16: 248-287.

Jacobson, R., 1960: Concluding Remark, in: Style and Language (hrsg. v. Th. Sebök), Cambridge/Mass.

Jones, M. O., 1987 (Hrsg.): Inside Organizations. Newbury Park: Sage.

Jupp, T. C./ C. Roberts/ J. Cook-Gumperz, 1982: Language and disadvantage: The hidden dimension, $S$. 232-256. In: Gumperz (Hrsg.), 1982b.

Kallmeyer, W. (Hrsg.), 1990: Kommunikation in der Stadt. Exemplarische Analysen des Sprachverhaltens in Mannheim. Berlin und New York: de Gruyter.
Kendon, A., 1990: Conducting Interaction. Patterns of Behavior in Focused Encounters. Cambridge.

Kloss, H., 1977: Über einige Terminologie-Probleme der interlingualen Soziolinguistik. Deutsche Sprache 3: 224-237.

Kochman, T. (Hrsg.), 1972, Rappin' and Stylin' Out: Communication in Urban Black America. Urban/Ill.

Levinson, S. C., 1979: Activity Types and Language. Linguistics 17: 356-389.

Lindenfeldt, J., 1984: De l'ethnographie de la communication à la sociolinguistique interactionniste. L'Homme XXIV, 131-135.

Luckmann, Th., 1985: Von der unmittelbaren zur mittelbaren Kommunikation. In: T. Borbe (Hrsg.), Mikroelektronik. Die Folgen für die zwischenmenschliche Kommunikation. Berlin.

Luckmann, Th., 1986: Grundformen der gesellschaftlichen Vermittlung des Wissens: Kommunikative Gattungen, S. 191-211. In: F. Neidhart, M. R. Lepsius, J. Weiß (Hrsg.), Kultur und Gesellschaft. Sonderband 27 d. KZfSS. Opladen.

Luckmann, Th., 1991: Einleitung zum Bericht des Forschungsprojektes „Rekonstruktive Gattungen der alltäglichen Komunikation. Ms. Konstanz.

Martin-Jones, M., 1989: Language, Power and Linguistic Minorities. Sociological Review Monographs 36.

Milroy, L., 1987: Language and Social Networks. Oxford (2. Aufl.).

Philipsen, G./ D. Carbaugh, 1986: A bibliography in the ethnography of communication. Language and Society 15: 387-398.

Quasthoff, U. M., 1981: Zuhöreraktivitäten beim konversationellen Erzählen, S. 287-313. In: P. Schröder/ H. Steger (Hrsg.), Dialogforschung. Düsseldorf.

Rehbein, J. (Hrsg.), 1985: Interkulturelle Kommunikation. Tübingen: Niemeyer.

Sanches, M./ B. Blount (Hrsg.), 1975: Sociocultural Dimensions of Language Use. New York.

Saville-Troike, M., 1984: The Ethnography of Communication. Oxford. (2. Aufl.).

Schmitz, H. W., 1975: Ethnographie der Kommunikation. Hamburg.

Schütz, A./ Th. Luckmann, 1979: Strukturen der Lebenswelt. Frankfurt.

Schütze, F., 1987: Die Rolle der Sprache in der soziologischen Forschung, S. 413-431. In: Ammon/Dittmar/ Mattheier (Hrsg.).

Sherzer, J., 1990: Verbal Art in San Blas. Kuna Culture Through its Discourse. Cambridge.

Streeck, J., 1985: Kulturelle Kodes und ethnische Grenzen. Drei Theorien über Fehlschläge der interethnischen Kommunikation, S. 103-120. In: J. Rehbein (Hrsg.), Interkulturelle Kommunikation. Tübingen. 